\title{
Screening for Prediabetes and Type 2 Diabetes Mellitus
}

\author{
Neda Laiteerapong, MD, MS and Adam S. Cifu, MD \\ University of Chicago, Chicago, Illinois.
}

\section{Summary of the Clinical Problem}

Abnormal blood glucose and type 2 diabetes are prevalent conditions in the United States; about 115 million adults have prediabetes or diabetes. ${ }^{1}$ Prediabetes is defined as IFG, IGT, or an increased average blood glucose level indicated by an increased hemoglobin $\mathrm{A}_{1 \mathrm{c}}$ level. The incidence of type 2 diabetes among patients with prediabetes may be as high as 35.5 to 70.4 new diagnoses per 1000 person-years. ${ }^{2}$ In 2014, type 2 diabetes affected $9.3 \%$ of US adults aged 20 years or older, and $36.4 \%$ of adults with type 2 diabetes were undiagnosed. ${ }^{1}$ Because early-stage type 2 diabetes is often asymptomatic, diagnosis can be delayed commonly from 4 to 8 years. ${ }^{3}$ Abnormal blood glucose and type 2 diabetes can be screened for by well-established tests. Type 2 diabetes significantly increases the risk of several comorbid conditions; eg, kidney failure, nontraumatic lower limb amputations, blindness, heart disease, stroke, peripheral vascular disease, dementia, and depression. Treatments to maintain euglycemia include lifestyle changes, medication therapies, and surgery.

\section{Characteristics of the Guideline Source}

This guideline ${ }^{4}$ was written by the USPSTF, an independent panel of national experts in prevention and evidence-based medicine (Table). This research was funded by AHRQ, whose staff worked with USPSTF members to develop and refine the scope, framework, and questions. AHRQ played no role in study selection, quality assessment, synthesis, or development of conclusions. AHRQ provided project oversight and reviewed the draft and final reports to ensure that they met methodological standards. USPSTF members are from the fields of preventive medicine and primary care, including internal medicine, family medicine, behavioral health, and nursing. USPSTF considers the benefits and harms of medical services but not the costs of providing the services. A draft of the recommendations was posted for comment on the USPSTF website before publication. Potential financial, professional, and intellectual conflict-of-interest disclosures were completed by all members.

Corresponding Author: Adam Cifu, MD, University of Chicago, 5841 S Maryland Ave, MC 3051, Chicago, IL 60637

(adamcifu@uchicago.edu).

Conflict of Interest Disclosures: The authors have completed and submitted the ICMJE Form for Disclosure of Potential Conflicts of Interest and none were reported.

Related Guidelines

ADA Standards of Medical Care in Diabetes-2015

Consensus Statement by the AACE and American College of Endocrinology on the Comprehensive Type 2 Diabetes Management Algorithm—2016 Executive Summary 


\section{Evidence Base}

The 2015 clinical guideline is an update of a 2008 USPSTF recommendation on screening for abnormal blood glucose and type 2 diabetes mellitus. In 2008, the USPSTF recommended that asymptomatic adults with hypertension (defined as sustained blood pressure of $>135 / 80 \mathrm{~mm} \mathrm{Hg}$ ) should be screened for diabetes (grade B recommendation). The current guideline is based on a review of studies from the prior USPSTF guidelines; relevant references from the Cochrane Central Register for Controlled Trials, the Cochrane Database of Systematic Reviews, and MEDLINE (2007-October 2014); and reference lists of relevant articles. ${ }^{5}$ The review focused on the benefits and harms of screening for prediabetes or diabetes, interventions for screen-detected abnormal blood glucose, and treatment of IFG or IGT. USPSTF also examined evidence of differences in the effects of screening and interventions by subpopulation and of the benefits and harms of treating other cardiovascular risk factors (aspirin use and intensive vs standard control of blood glucose, blood pressure, and lipid levels in adults with diabetes).

\section{Benefits and Harms}

In conjunction with development of this guideline, asystematic review was conducted. ${ }^{5}$ There is no evidence that screening for diabetes decreases clinical outcomes, and in fact there is some evidence that it does not decrease outcomes. Two trials randomizing patients to screening or no screening for diabetes found no differences in mortality. An important presumed benefit of screening for abnormal blood glucose would be delay or prevention of diabetes when screening is paired with lifestyle interventions for overweight or obese individuals. Sixteen trials found that treatment of IFG or IGT delayed or prevented progression to diabetes. This was true with lifestyle interventions (pooled RR from 10 trials, 0.53 ; 95\% CI, 0.39-0.72) or medications, including metformin plus rosiglitazone (number needed to treat over 3.9 years, 4 ; 95\% CI, 2.7-7.1), thiazolidinediones (pooled RR from 2 studies, 0.42; 95\%CI,0.37-0.47), and a-glucosidase inhibitors (pooled RR from 4 studies, $0.64 ; 95 \% \mathrm{CI}, 0.45-0.90]$ ). However, no significant reductions in all-cause mortality (pooled odds ratio, $1.01 ; 95 \% \mathrm{CI}, 0.87-1.18$ ) or cardiovascular mortality (pooled odds ratio, 1.06; 95\% CI, 0.84-1.35) were found after treating screen-detected or early diabetes, IFG, or IGT with pharmacologic interventions or in combination with lifestyle modification in most studies. One trial with much longer follow-up (23 years vs $<10$ years) did find that a 6-year lifestyle intervention reduced the risk of all-cause mortality (HR, 0.71; 95\% CI, 0.51-0.99) and cardiovascular mortality (HR, 0.59; 95\% CI, 0.36-0.96) after 23 years of follow-up. ${ }^{6}$

Little harm is to be expected from screening for abnormal blood glucose. The only potential harm is an increase in short-term (6-week) but not long-term anxiety. ${ }^{7}$ Subsequent potential harms included adverse effects related to drug therapy for adults who screened positive for prediabetes.

\section{Discussion}

This USPSTF guideline stems from a careful and thorough review of the evidence. These guidelines are consistent with 2 other USPSTF recommendations advising intensive 
behavioral interventions: a 2012 recommendation supporting screening for obesity and offering or referring patients with obesity for intensive behavioral interventions and a 2014 recommendation suggesting offering or referring patients who are over-weight or obese and have 1 cardiovascular risk factor for intensive behavioral interventions. These guidelines reinforce the importance of health behaviors in the development of chronic diseases and the opportunity to improve outcomes by improving lifestyle choices.

Full implementation of these guidelines will likely be challenged by the limited reimbursement and availability of intensive behavioral interventions. In 2011, intensive behavioral therapy for obesity did become a covered preventive service by Medicare Parts A and B. From a societal perspective, the intervention is probably cost-effective and possibly cost-saving, depending on the cost of delivery, number of sessions provided, and effectiveness of the intervention sessions. ${ }^{8}$ However, from a health system perspective, reimbursement for 15 -minute visits is low (approximately $\$ 25 /$ session), and it is unclear if providing intensive behavioral therapy services is sustainable for clinics. It is likely that intensive behavioral health services will be limited to larger health care centers that are more able to absorb the risk of providing this covered service.

Current evidence of clinical benefit for screening for prediabetes and diabetes is scant. Most current studies have short follow-up periods, which are unlikely to show reductions in clinical end points because the gap between initiating intensive glycemic treatment and improved microvascular outcomes may be a decade; for cardiovascular outcomes and mortality, it may be 2 decades. ${ }^{9,10}$ If reductions in clinical end points exist, the number needed to screen is likely large.

The USPSTF guidelines recommend initiating screening at a slightly younger age than the American Diabetes Association (ADA) guidelines (40 years vs 45 years) and provide an age at which to stop screening (on which the ADA and the American Association of Clinical Endocrinologists [AACE] do not comment). The AACE does not recommend screening based on age. Both the ADA and AACE recommend screening based on clinical risk factors, similar to those included in these guidelines.

\section{Areas in Need of Future Study or Ongoing Research}

Studies that examine the effects of screening large populations for abnormal blood glucose on hard outcomes, like mortality, are necessary. The chronic nature of diabetes necessitates that these studies provide long-term follow-up to understand the lifetime consequences of population-level screening. Also, it is unclear if the effects of screening differ by age, sex, race, or ethnicity. Because older adults and racial/ethnic minorities share a disproportionate burden of diabetes, it would be reasonable to assume that they may benefit from earlier screening onset and/or decreased screening interval. Additional studies need to conclusively determine the frequency and age of initiation for screening for abnormal blood glucose in these subpopulations. 


\section{References}

1. Centers for Disease Control and Prevention. [Accessed October 29, 2015] National Diabetes Statistics Report. 2014. http://www.cdc.gov/diabetes/data/statistics/2014statisticsreport.html

2. Morris DH, Khunti K, Achana F, et al. Progression rates from $\mathrm{HbA}_{1 \mathrm{c}} 6.0-6.4 \%$ and other prediabetes definitions to type 2 diabetes. Diabetologia. 2013; 56(7):1489-1493. [PubMed: 23584433]

3. Samuels TA, Cohen D, Brancati FL, et al. Delayed diagnosis of incident type 2 diabetes mellitus in the ARIC study. Am J Manag Care. 2006; 12(12):717-724. [PubMed: 17149994]

4. Siu AL. USPSTF. Screening for abnormal blood glucose and type 2 diabetes mellitus. Ann Intern Med. 2015; 163(11):861-868. [PubMed: 26501513]

5. Selph S, Dana T, Blazina I, et al. Screening for type 2 diabetes mellitus. Ann Intern Med. 2015; 162(11):765-776. [PubMed: 25867111]

6. Li G, Zhang P, Wang J, et al. Cardiovascular mortality, all-cause mortality, and diabetes incidence after lifestyle intervention for people with impaired glucose tolerance in the Da Qing Diabetes Prevention Study. Lancet Diabetes Endocrinol. 2014; 2(6):474-480. [PubMed: 24731674]

7. Park P, Simmons RK, Prevost AT, Griffin SJ. Screening for type 2 diabetes is feasible, acceptable, but associated with increased short-term anxiety. BMC Public Health. 2008; 8:350. [PubMed: 18840266]

8. Hoerger TJ, Crouse WL, Zhuo X, et al. Medicare's intensive behavioral therapy for obesity. Am J Prev Med. 2015; 48(4):419-425. [PubMed: 25703178]

9. Holman RR, Paul SK, Bethel MA, et al. 10-year follow-up of intensive glucose control in type 2 diabetes. N Engl J Med. 2008; 359(15):1577-1589. [PubMed: 18784090]

10. UK Prospective Diabetes Study Group. Effect of intensive blood-glucose control with metformin on complications in overweight patients with type 2 diabetes (UKPDS 34). Lancet. 1998; 352(9131):854-865. [PubMed: 9742977] 


\section{MAJOR RECOMMENDATIONS}

Screen for glucose abnormalities every 3 years. Use hemoglobin $\mathrm{A}_{1 \mathrm{c}}$ level, fasting plasma glucose level, or oral glucose tolerance test. Consider screening at a younger age or at a lower body mass index in adults with a family history of diabetes, with a history of gestational diabetes or polycystic ovary syndrome, or who are members of racial/ethnic minority groups. Adults who screen positive for impaired fasting glucose (IFG), impaired glucose tolerance (IGT), or type 2 diabetes should be offered or referred to intensive behavioral counseling interventions to improve their diet and increase physical activity (B recommendation for all of the above). 


\section{Table}

\section{Guideline Rating}

\begin{tabular}{|ll|}
\hline Rating Standard & Rating \\
\hline 1. Establishing transparency & Good \\
\hline 2. Management of conflict of interest in the guideline development group & Good \\
\hline 3. Guideline development group composition & Fair \\
\hline 4. Clinical practice guideline-systematic review intersection & Good \\
\hline 5. Establishing evidence foundations and rating strength for each guideline recommendations & Good \\
\hline 6. Articulation of recommendations & Good \\
\hline 7. External review & Good \\
\hline 8. Updating & Fair \\
\hline 9. Implementation issues & Good \\
\hline
\end{tabular}

